This item was submitted to Loughborough's Institutional Repository (https://dspace.lboro.ac.uk/) by the author and is made available under the following Creative Commons Licence conditions.

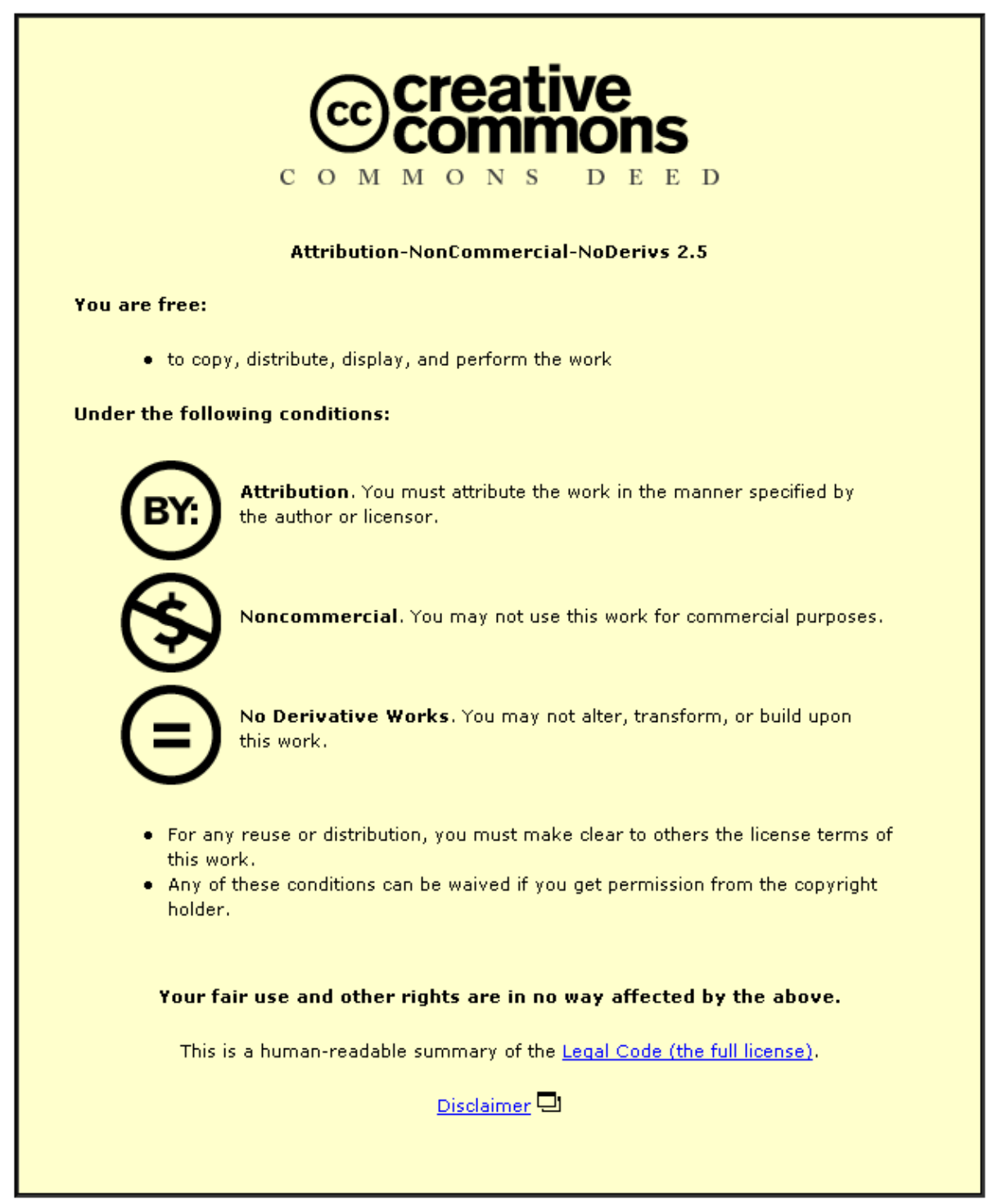

For the full text of this licence, please go to: http://creativecommons.org/licenses/by-nc-nd/2.5/ 


\title{
Simulation of incoherent and coherent backscattered wave fields from cavities in a solid matrix
}

\author{
Valerie J. Pinfield ${ }^{\text {a) }}$ and Richard E. Challis \\ Electrical Systems and Optics Division, Faculty of Engineering, University of Nottingham, University Park, \\ Nottingham, NG7 2RD, United Kingdom
}

(Received 17 November 2011; revised 12 September 2012; accepted 3 October 2012)

\begin{abstract}
This paper reports a study of the backscattered ultrasonic signal from a solid layer containing spherical cavities, to determine the conditions in which an effective medium model is a valid description of the response. The work is motivated by the need to model the response of porous composite materials for ultrasonic non-destructive evaluation (NDE) techniques. The numerical simulation predicts the response of a layer containing cavities at a single set of random locations, and compares it to the predicted response from a homogeneous layer with ensemble-averaged material properties (effective medium model). The study investigates the conditions in which the coherent (ensembleaveraged) response is obtained even from a single configuration of scatterers. Simulations are carried out for a range of cavity sizes and volume fractions. The deviation of the response from effective medium behavior is modeled, along with the trends as a function of cavity radius, volume fraction, and frequency, in order to establish an acceptability criterion for application of an effective medium model. (C) 2012 Acoustical Society of America. [http://dx.doi.org/10.1121/1.4763985]
\end{abstract}

PACS number(s): 43.35.Cg, 43.35.Zc [PEB]

Pages: $3760-3769$

\section{INTRODUCTION}

Ultrasonic or acoustic propagation through inhomogeneous media is of relevance in a wide variety of fields, from seismology, food and pharmaceutical applications, and nondestructive evaluation. ${ }^{1}$ Considering, in particular, materials with inclusions, which may be fibers in a resin, cavities in a cast metal, particles in a suspension, etc., the characteristics of compressional or shear waves through such materials are of great interest. Measurements of sound speed and attenuation, for example, can be used for dispersed phase particle sizing or the characterization of porosity. The detection of defects in fiber composites relies on knowledge of the sound speed in the composite. Accordingly a vast literature exists on the determination of the properties of such media pertinent to ultrasonic propagation.

In the majority of studies, what is considered is the "coherent field," that which propagates "uniformly" as if in a homogeneous medium, and can thus be characterized by a wavenumber, acoustic impedance, or by properties such as elastic moduli and density. ${ }^{2-6}$ For this part of the wave field, therefore, the material can be defined by effective properties corresponding to an equivalent homogeneous medium with the same ultrasonic propagation characteristics. Many different schemes exist for the derivation of these effective properties, including models termed effective medium models, self-consistent models, multiple scattering models, ensemble-average models, and homogenization schemes. A broad selection of such studies was reviewed in a previous paper; ${ }^{7}$ papers by Kanaun and Levin, ${ }^{8}$ Kim, ${ }^{5}$ and Parnell et al. ${ }^{6}$ also include useful reviews of this literature. The field

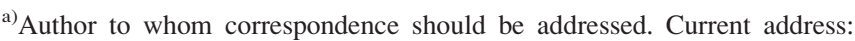
Chemical Engineering Department, Loughborough University, Loughborough, UK, LE11 3TU. Electronic mail: v.pinfield@lboro.ac.uk
}

is still an active one, despite its long history, and although many works are based on elastostatic analyses ${ }^{9,10}$ rather than on wave propagation, new variants on effective medium models are published frequently. ${ }^{11,12}$

Let us consider what this coherent field is, and whether it is indeed what is actually measured in an experimental system. Foldy ${ }^{13}$ states that although the scattered fields are all coherent, the resultant field is separated into that part termed (and now known as) the coherent field which propagates uniformly and other scattered components referred to as incoherent scattering. It is the identification of that coherent field component that leads to the requirement to obtain the ensemble average of the scattered fields, that is the average over all possible configurations of scatterer positions. ${ }^{2}$ Such ensemble-averaging is a principal component of many effective property derivations, particularly those based on the multiple scattering formulation. ${ }^{2,6,13-16}$

Hence, the effective properties derived from such formulations correspond to an average over all possible scatterer configurations. In practice, however, is a measurement taken on a sample similarly averaged over all configurations of scatterer locations? Waterman and Truell, ${ }^{14}$ in their multiple scattering model for the effective wavenumber, state that this averaging process occurs naturally in many applications "either due to the measuring device averaging over a region large compared with any of the lengths involved, or where the configurations are changing with time rapidly in comparison with the time scale of measurement." While the latter is certainly true for measurements in fluid systems, the locations of scatterers in a solid (whether cavities or inclusions) are fixed. The former requirement implies use of spatial averaging over a volume of sample sufficiently large to smooth any statistical variations in scatterer distribution; how large is not established. A similar spatial averaging effect, by taking measurements on a number of samples, is 
suggested by Foldy ${ }^{13}$ in order to improve the averaging over scatterer configurations, a requirement also confirmed by Dubois et al. ${ }^{17}$ using measurements at different positions on the same sample. In continuum mechanics, the question of how large a sample volume is required to approach statistical homogeneity of mechanical properties has been considered by Ostoja-Starzewski and others. ${ }^{18,19}$ In these studies, the statistically homogeneous sample (theoretically relating to an infinite set of microscale samples) is referred to as the Representative Volume Element (RVE), while a finite-sized sample with specific microstructure is termed the Statistical Volume Element (SVE). The approach of the SVE to the RVE explored by Ostoja-Starzewski is similar in nature to the sampling question addressed in the present work.

For a solid system, then, an average over configurations could be achieved by averaging measurements across a large transducer surface, or taken at different positions, or on different samples, thus averaging over many configurations. However, in non-destructive evaluation (NDE) applications, there is a requirement to identify the characteristics of the material at a particular location (although this still corresponds to a finite volume element) which precludes the use of averaging over a large area. Therefore a measurement is made with a single local configuration of scatterer locations, occurring in the relevant measurement region. Foldy ${ }^{13}$ is apparently unconcerned by the use of a measurement from a single configuration of scatterer locations, stating that "...for a collection of a large number of scatterers a particular 'unprepared' collection will have particular physical properties which do not deviate greatly from the average physical properties of a properly defined statistical ensemble of collections because of the lack of 'correlation' implied in the word 'unprepared' as to the positions of the individual scatterers. This allows one to estimate the properties of the average over an ensemble from an experiment on a particular configuration with a very high probability that the estimate is correct, although the possibility exists of a wide deviation if the selected ensemble should be particularly strongly ordered." This present study aims to investigate whether the signal backscattered from a region of scatterers with only a single realization of locations can be represented by the ensemble average result.

In a previous paper, ${ }^{7}$ numerical simulations were used to demonstrate that a simple numerical ensemble-average limit of the scattered fields from a layer of spherical scatterers was equivalent to the field obtained by considering the layer as homogeneous with certain effective properties. In addition, the simulations showed that, under certain conditions, the field generated by scatterers in a single configuration of scatterer locations was also equivalent to the effective medium response. In the present work, further simulations are reported which demonstrate the conditions under which the response of a single realization of scatterer locations is similar to that of the ensemble-averaged effective medium. This particular aspect has not been addressed in the literature to our knowledge. Simulations of ensemble-average responses have, however, been reported, and since these generate the response from many scatterer configurations, they do in some cases provide useful information on the current prob- lem. Maurel ${ }^{20}$ constructed a one-dimensional simulation to validate an ensemble average model, taking averages of between 100 and 10000 realizations of scatterer locations. The results indicated that a greater number of realizations were required to achieve convergence of the average for larger wavenumbers (shorter wavelength), and that considerably more realizations were required to obtain the required accuracy for the reflected fields than for the transmitted coherent field. The large number of realizations required would suggest that the results of a single realization can be significantly far from the ensemble average result; indeed a selection of responses is shown for single realizations, demonstrating the incoherent field contribution. ${ }^{20}$ The deviation is expected to be greater for shorter wavelengths where more realizations were required to achieve convergence of the average field. ${ }^{20}$

Two finite-difference time-domain simulations have been reported, both simulating solid cylinders in a fluid in two dimensions. ${ }^{17,21}$ Dubois et al. ${ }^{17}$ found that acceptable convergence of the averaged transmitted field was obtained using around 30 realizations of scatterer locations, although, similar to Maurel, ${ }^{20}$ they found that the reflected field converged more slowly, requiring a much greater number (90 to 210) of simulations. Their calculations covered a broad range of ratios of wavelength to radius, using two different concentrations, and represented a similarly broad range of ratio of wavelength to the mean distance between scatterers. For most of the simulations, the mean distance between scatterers would be less than or comparable to the wavelength. Only ensemble averaged results are presented in the paper, no single realization results are shown. Galaz et al., ${ }^{21}$ using a similar system, accept an inaccuracy of around $9 \%$ on attenuation by using only 15 simulations to calculate the averaged field (due to computational time constraints). The authors state that the standard deviation of the results from individual realizations are considerably higher than this figure, which indicates that use of a single realization could in practice be far from the averaged result. Although the authors were again interested in the averaged values, one simulation of a single realization is given, which clearly shows the effect of the incoherent field on the reflected (backscattered) signal.

In the works of both Dubois et al. ${ }^{17}$ and Galaz et al. ${ }^{21}$ the simulated receiving surface is much larger than the scatterer radius, and much larger also than the mean distance between scatterers. This would suggest that the averaging effect of the finite transducer area does not achieve the convergence of the scattered field to the ensemble averaged result until a very large area is covered. In order to achieve the same averaging effect as the use of multiple simulations, a scale-up factor on the receiver area on the order of the number of simulations (realizations) would be required. In all the simulations reported, ${ }^{17-21}$ the wavelength is comparable to or less than the mean distance between scatterers, although Dubois et al. also cover a small range in which the wavelength is much greater than the mean distance between scatterers.

The simulations reported in this present work address the convergence of the backscattered response from a single realization of spherical scatterers in a solid matrix to the 
ensemble-averaged response. We wish to establish the conditions under which the response from a layer of cavities can be described by an effective medium model, without resort to spatial averaging. Our models are based on a scattering formulation originating in the work of Rayleigh, ${ }^{22}$ both for the single realization simulations and for the effective medium (ensemble-averaged) responses. The scattered field from a randomly-generated single configuration of spherical cavities is simulated using a model, termed the discrete scatterer model (Sec. II B, Fig. 1, top half), demonstrating the emergence of the coherent field behavior from the incoherent scattered fields. The response is then compared to that of the ensemble average limit of the discrete scatterer model (Sec. II B), and an effective medium model (Sec. II C) which simulates reflection from a homogeneous layer (Fig. 1, bottom half), whose properties are derived from the Foldy ensemble-averaged wavenumber. The work extends that reported in the previous paper ${ }^{7}$ by investigating the effects of cavity radius, concentration, and frequency to establish the validity criteria for effective medium properties applied to single scatterer configurations. Fuller details of the models were given in the previous paper $^{7}$ and are only summarized here. The results of the models are shown in Sec. IV, and the conditions for validity of the effective medium description for the field scattered from a single configuration of scatterers are discussed in Sec. V.

\section{THE MODELS}

\section{A. Discrete scatterer model}

A number of spherical cavities are located in the region $z_{\min } \leq z \leq z_{\max }$, irradiated by a longitudinal plane wave of infinite lateral extent transmitted by an infinite planar transducer coupled directly to the solid medium (top half of Fig. 1). The acoustic field scattered by the cavities is received at the transducer, which is assumed to respond to the normal displacement at its face. The signal received at a point on the transducer is derived by considering the field scattered by each cavity, taking into account its angular dependence in the

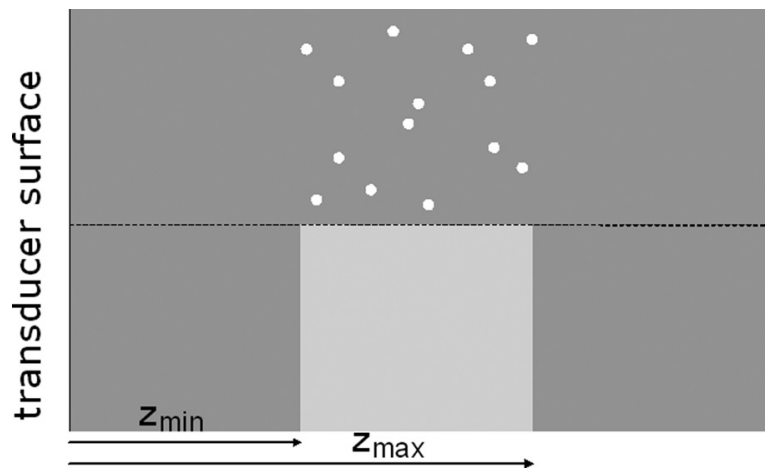

FIG. 1. System configurations for the two models. The top half, above the dashed line, shows the discrete scatterer model with spherical cavities embedded in solid material in the region $z_{\min }<z<z_{\max }$. The bottom half, below the dashed line, shows the effective medium model in which a homogeneous solid material is present in that region. In both cases, the transducer is directly in contact with the medium in the region $z<z_{\min }$ which has the same properties as the matrix surrounding the cavities, and the material in the region $z>z_{\max }$. far field. It should be noted that there are no planar interfaces between regions in this model; the matrix surrounding the cavities is identical to the medium between the transducer and the porous region, and to that behind the porous region. The wavenumber of this matrix material is $k=\omega / c(\omega)+i \alpha(\omega)$, where $c(\omega)$ and $\alpha(\omega)$ are the wave speed and the attenuation, respectively, and $\omega$ is the angular frequency. The transmitted wave is harmonic, and the $e^{-i \omega t}$ convention is adopted for time-dependence. For simplicity in the numerical simulation, the exciting wave at any scatterer is assumed to be identical to the incident wave; no modification of the incident wave is made to incorporate the scattered fields from other scatterers. This assumption limits the simulation to low concentrations but in order to establish the trends with concentration, calculations have been carried out up to concentrations of $10 \%$ by volume.

The normal displacement at a point on the transducer due to the scattered fields from the cavities is given by

$$
\begin{aligned}
\mathbf{u}_{z, \text { mult }}= & \mathbf{u}_{z, \text { inc }} \sum_{j=1}^{N_{s c}} e^{i k z_{j}} \cdot \frac{e^{i k r_{j}}}{r_{j}^{2}}\left[-\left.\frac{\partial f(\theta)}{\partial \theta}\right|_{\theta_{j}} \sin \theta_{j}\right. \\
& \left.-i k z_{j} \cdot f\left(\theta_{j}\right)\left\{1-\frac{1}{i k r_{j}}\right\}\right]
\end{aligned}
$$

where $\mathbf{u}_{z, \text { inc }}$ is the normal displacement due to the transmitted (incident) field at the same point on the transducer, $N_{s c}$ is the total number of scatterers (cavities), and the geometrical variables are defined in the diagram shown in Fig. 2. The far-field amplitude $f(\theta)$ is defined in terms of the displacement potential for the scattered field by

$$
\phi \rightarrow f(\theta) \frac{e^{i k r}}{r} \quad \text { as } \quad r \rightarrow \infty .
$$

According to the Rayleigh method, it is related to the scattering coefficients of the partial wave orders, $A_{n}$ as

$$
f(\theta)=\frac{1}{i k} \sum_{n=0}^{\infty}(2 n+1) A_{n} P_{n}(\cos \theta)
$$

where $P_{n}$ is the Legendre polynomial. In the present simulations, these scattering coefficients are calculated by the Rayleigh method, as formulated by Ying and Truell, ${ }^{23}$ described in Refs. 1 and 24. Only orders up to $n=2$ make a significant contribution except at the largest radius and highest frequencies studied, but calculations included up to $n=5$. Analytical expressions for the coefficients in the long wavelength limit were given in an earlier paper. ${ }^{7}$

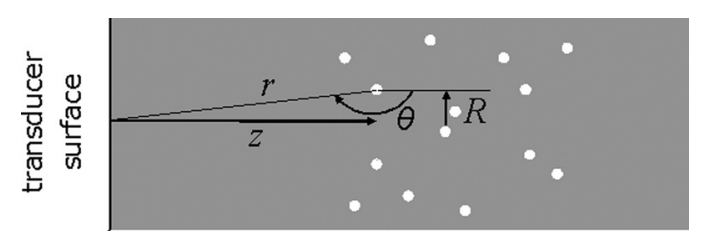

FIG. 2. Diagram showing the coordinate definitions for the location of a single cavity relative to the point under consideration on the transducer. 
Although in principle the region containing cavities is of infinite lateral extent, the contribution to the received signal decreases as the radial position $R$ of the cavity increases. Hence, it is possible to consider only a finite domain, $z_{\min } \leq z \leq z_{\max }, R \leq R_{\max }$ within which cavities are located. The value of $R_{\max }$ for the simulations is determined such that a further increase in $R_{\max }$ results in no significant change in the received signal. Since the simulation covers a large lateral region, some effective averaging over scatterer locations may already occur. Scatterers are placed randomly in the region, with no account taken of their excluded volume, although their scattering properties are defined by a denoted scatterer radius. This is equivalent to the assumption of an uncorrelated distribution of scatterers, which is common in the effective medium literature. ${ }^{20,25}$

Equation (1) defines the Discrete Scatterer Model, for a single realization of scatterer (cavity) locations, and for a particular concentration of cavities and cavity size.

\section{B. Ensemble average model}

In common with many effective medium models, we take the ensemble average of the backscattered field from a single realization of scatterers to estimate the coherent field. Assuming uncorrelated scatterer locations, neglecting excluded volume (see above), the ensemble average field is given by

$$
\mathbf{u}_{z, \text { ave }}=\int_{z=z_{\min }}^{z_{\max }} \int_{r=z}^{\infty} 2 \pi N \mathbf{u}_{z, \text { sgle }} r d r d z
$$

where $N$ is the number density of scatterers. It should be noted that this ensemble averaged response is obtained under a single-scattering assumption, with the same incident field at each scatterer. It does not correspond exactly to the coherent field derived from a multiple scattering model in which the exciting field at each scatterer includes the scattered fields from all other scatterers. It is, however, the ensemble average limit of our discrete scatterer model which is a single scattering model. These simplifications have been made in order to achieve simulations of a large system.

\section{Effective medium model}

In contrast to the discrete scatterer model described above, the effective medium model treats the region containing cavities as an equivalent homogeneous medium (Fig. 1, bottom half). The properties of this region are derived from published analytical ensemble-average models for the effective density, ${ }^{4,25,26}$ and wavenumber, ${ }^{13}$ and so the model produces the coherent field response. There are three regions of homogeneous material, with planar interfaces at $z=z_{\min }$ and $z=z_{\max }$. The properties of the material in the regions $z<z_{\min }$ and $z>z_{\max }$ are identical to those of the matrix surrounding the cavities, as in the previous model. However, the region $z_{\min }<z<z_{\max }$ is now considered as homogeneous with effective properties and with no actual cavities present.
The normal displacement at the transducer can be obtained by summing reflections from the front and back interfaces in a straightforward manner, leading to

$$
\begin{aligned}
\mathbf{u}_{z, \text { eff }}(\omega)= & \mathbf{u}_{z, \text { inc }} e^{2 i k z_{\min }} \cdot r_{12} \\
& \times\left[1-t_{12} t_{21} e^{2 i k d}\left\{1-r_{12} e^{2 i k d}\right\}^{-1}\right],
\end{aligned}
$$

where $d=z_{\max }-z_{\min }$ is the thickness of the layer and $r_{i j}$ and $t_{i j}$ are the displacement reflection and transmission coefficients at the interface from medium $i$ to medium $j$, which are defined by

$$
r_{12}=-\frac{\hat{Z}-1}{\hat{Z}+1}, \quad t_{12}=\frac{2 \hat{Z}}{\hat{Z}+1} \text { and } t_{21}=\frac{2}{\hat{Z}+1},
$$

where $\hat{Z}$ is the ratio of the impedance in the layer to that of the solid matrix.

The impedance of a medium is given by

$$
Z=\rho c,
$$

with density $\rho$ and longitudinal sound speed, $c$. The effective density is

$$
\rho_{\text {eff }}=\rho(1-\phi)
$$

where $\phi$ is the fractional volume occupied by the cavities. This result has been obtained by a number of workers using ensemble average schemes, ${ }^{4,25,26}$ or alternative homogenization methods. ${ }^{6}$ The wave speed is also obtained from an ensemble averaged scattered field; here we adopt the result of Foldy ${ }^{13}$ which is first order in concentration, which leads to

$$
\left(\frac{K^{2}}{k^{2}}\right)=1+\frac{3 \phi}{k^{2} a^{3}} f(0),
$$

for the effective, ensemble-averaged wavenumber, $K$, and $k$ is the wavenumber in the matrix. The effective wave speed results from the definition of the wavenumber as $K=\omega / c_{\text {eff }}+i \alpha_{\text {eff }}$. It can be shown that the resulting impedance ratio for the effective medium is independent of frequency in the low frequency region.

\section{NUMERICAL CALCULATIONS}

\section{A. System parameters}

All calculations have been executed in MATLAB ${ }^{27}$ using double precision complex arithmetic. Simulations using the discrete scatterer model were conducted for a set of realizations of cavity locations. A range of cavity radii from 5 to $20 \mu \mathrm{m}$ was used, with all cavities in a single realization of scatterer locations being the same size. For each selected cavity size, a single set of cavity locations is generated randomly within the defined region $(2 \mathrm{~mm}<z<3 \mathrm{~mm})$, to represent a concentration of $20 \%$ by volume. Excluded volume effects were not accounted for. Cavity locations, specified by independent coordinates $z, R$, were obtained using the MATLAB pseudo-random number generator in a manner which 
TABLE I. The system parameters used in the calculations.

\begin{tabular}{ll}
\hline \hline Distance of layer from transducer $z_{\min }$ & $2 \mathrm{~mm}$ \\
Layer thickness & $1 \mathrm{~mm}$ \\
Radius of porous region $R_{\max }$ & $20 \mathrm{~mm}$ \\
Cavity volume fractions & $1 \%, 2 \%, 5 \%, 10 \%$ \\
Cavity radius & $5.0,7.9,10.0,15.9,20.0 \mu \mathrm{m}$ \\
Number of cavities (millions) in a region at & \\
20\% concentration (respectively with radius) & $480,120,60,15,7.5$ \\
Transducer center frequencies & $10 \mathrm{MHz}, 5 \mathrm{MHz}$ \\
Sampling frequency & $50 \mathrm{MHz}$ \\
Number of samples & 1024 \\
\hline \hline
\end{tabular}

preserved a constant probability of scatterers per unit volume. Lower concentrations are obtained using a subset of that location set. In this way, a set of scattered signals is generated for each cavity size and concentration. The system parameters are shown in Table I.

Calculations were discretised in the time and frequency domains, with a sampling frequency of $50 \mathrm{MHz}$ and a record length of 1024 samples. Frequency-domain simulations based on the models were combined with typical transducer signals and transformed into the time-domain by Fourier transform. The signal used as input to the simulations was that obtained experimentally using a pair of identical transducers of $10 \mathrm{MHz}$ center frequency (c.f.) (V311-SU, Olympus NDT, Waltham, MA) in a pitch-catch arrangement with $25 \mathrm{~mm}$ path length through water. The transducer waveform was digitized initially at $400 \mathrm{MHz}$ using a LeCroy 9450A oscilloscope (LeCroy Corp., Chestnut Ridge, NY) and then sub-sampled down to the simulation sampling frequency of $50 \mathrm{MHz}$. An additional simulated transducer transmitreceive response with a center frequency of around $5 \mathrm{MHz}$ was obtained by sub-sampling the measured $(10 \mathrm{MHz}$ c.f.) response in the frequency domain. All time-domain figures have been smoothed using an up-sample to $200 \mathrm{MHz}$; they are also time-shifted so that the temporal origin occurs at the first received signal at $2 z_{\mathrm{min}} / c$. In addition, scaling by concentration has been applied for the purposes of graphical comparison.

The solid matrix surrounding the cavities is taken to be a homogeneous representation of a typical carbon fiber reinforced composite, whose effective properties have been estimated as shown in Table II; the ratio of longitudinal:shear wave speed is taken to be $2: 1$. Attenuation in the composite is neglected in the present calculations so that the scattering processes and effective medium behavior could be investigated independently of the additional effects of frequencydependent losses in the resin matrix. Since we are considering the scattering problem in the long wavelength limit, the order $n$ of the partial waves can be limited and we have set

TABLE II. Physical properties of the composite matrix materials used in calculations.

\begin{tabular}{lc}
\hline \hline Sound speed (longitudinal) & $3035 \mathrm{~m} \mathrm{~s}^{-1}$ \\
Density & $1564 \mathrm{~kg} \mathrm{~m}^{-3}$ \\
Shear modulus & $3.6 \mathrm{GPa}$ \\
Attenuation & 0 \\
\hline \hline
\end{tabular}

$n_{\max }=5$, although only up to $n=2$ makes a significant contribution except at the largest radius and frequency. Here the wavelength at $10 \mathrm{MHz}$ is $\sim 300 \mu \mathrm{m}$ and the largest cavity radius $20 \mu \mathrm{m}$.

\section{B. Method of quality of fit}

Since the purpose of the present study is to explore the conditions under which a discrete scatterer simulation approaches that of a homogeneous layer in the ensemble average limit, it is necessary to establish criteria for the quality of fit in the comparisons between the model results. In each case, the comparison is between a discrete scatterer simulation and the corresponding effective medium or ensemble average reference signal. A number of comparison techniques were attempted in both time and frequency domains. The quality of fit calculations in the frequency domain were conducted on the system frequency response, without any transducer signal. Ultimately, calculations in the frequency domain were selected over time-domain methods on the basis that they permitted the effect of bandwidth to be established. Although other correlation techniques were again applied, the preferred measure was the sum of squared residuals (or residual sum of squares, RSS) on the absolute value of the frequency response, between the discrete scatterer response and the corresponding reference response, determined as a function of frequency.

The frequency response was first smoothed by windowing the corresponding time-domain response with a half-wave cosine from 5.31 to $5.84 \mu$ s after the expected first arrival of the front face reflection This effectively truncates the response before the arrival of the edge wave resulting from the boundary of the region containing cavities, and smooths the response from scatterers near that edge toward zero. The RSS, as defined here, is a measure of the deviation of a single realization response from the reference response, summed over frequency up to a given maximum frequency, and taken as a proportion of the reference response. As such it is effectively an estimate of the error of the single realization response as a fraction of the coherent reference response. Thus, at a frequency $f$, corresponding to sample number $l_{\max }$

$$
\operatorname{RSS}(f)=\frac{\sum_{l=1}^{l_{\max }}\left[\left|F_{\text {disc }, \phi, r}\left(f_{l}\right)\right|-\left|F_{\text {ref }, \phi, r}\left(f_{l}\right)\right|\right]^{2}}{\sum_{l=1}^{l_{\max }}\left|F_{\text {ref }, \phi, r}\left(f_{l}\right)\right|^{2}}
$$

where $F\left(f_{l}\right)$ is the frequency response at the discrete frequency $f_{l}$ denoted by the element integer $l=1+f_{l} n_{s} / f_{s}$, with $n_{s}$ the number of samples in the frequency response, and $f_{s}$ the sampling frequency. The subscripts "disc" and "ref" denote the discrete scatterer and reference (ensemble average, model B, or effective medium, model $\mathrm{C}$ ) responses, respectively, and subscripts $\phi$ and $r$ identify the response at a particular concentration and cavity radius.

Since a coherent response might be expected from a single realization of scatterers when the wavelength is much greater than the length scale of the inhomogeneity in the 
system, we introduce the mean distance between scatterers, $d$, estimated by the Wigner-Seitz radius, as a measure of that length scale, thus

$$
d=a / \phi^{1 / 3} \text {. }
$$

Then the parameters $k a$ and $k d$ can be used as (proportional) measures of the ratios of radius to wavelength and interscatterer distance to wavelength, respectively.

\section{RESULTS}

\section{A. Time-domain comparisons}

Plots of the simulated received signal according to the three models are shown in Fig. 3 for a variety of system parameters. In each figure, the reference signals (from the effective medium and ensemble average models) are compared with the corresponding discrete scatterer model result for a single realization of scatterer positions with the same cavity radius, concentration, and transducer center frequency. All signals have been scaled by concentration for the purpose of comparison in the figures. In each sub-figure, the effective medium received signal has separate parts corresponding to reflections from the front and back faces of the layer; the former being inverted, the latter not. This is characteristic of the response from a homogeneous layer. The ensemble average model also shows this layer-like characteristic. However, whereas the speed of the coherent wave changes with concentration, thus shifting the effective medium reflected signal, the ensemble average response (which uses a single-scattering assumption) takes the speed of the solid matrix and is therefore unaffected by concentration. The difference between the two reference signals is small at low concentrations but becomes significant at higher concentrations [see, for example, Fig. 3(b)].

Figure 3(a) shows the results for a $10 \mathrm{MHz}$ center frequency transducer, with a $15.9 \mu \mathrm{m}$ cavity radius at a concentration by volume of $2 \%(k a=0.3, k d=1.2)$. The only region where there is a similarity between the discrete scatterer model and the two reference models is for the first peak of the received signal; elsewhere, the discrete scatterer model, while largely retaining the oscillatory nature of the transmitted signal, varies greatly and apparently randomly in amplitude. Thus the incoherent field is significant in this case. The duration of the received signal is also much longer than for the effective medium and ensemble average models. In contrast, for a $5 \mathrm{MHz}$ center frequency, $5 \mu \mathrm{m}$ cavity radius and $2 \%$ concentration [Fig. 3(d)], the three models predict very similar results $(k a=0.05, k d=0.2)$. The discrete scatterer model approaches the ensemble average response, which is slightly time-shifted compared to the full coherent field from the effective medium model, due to the change in effective speed.

Comparing Figs. 3(a) and 3(b) shows that a higher concentration of $10 \%$ [Fig. 3(b)], with $k d=0.7$, brings the frontwall echo into close agreement, and reduces the amplitude of oscillations for the rest of the period. Figures 3(a) and 3(c) $(k a=0.1, \quad k d=0.4)$ show that a smaller cavity radius, with a smaller $k d$ value, similarly improves the
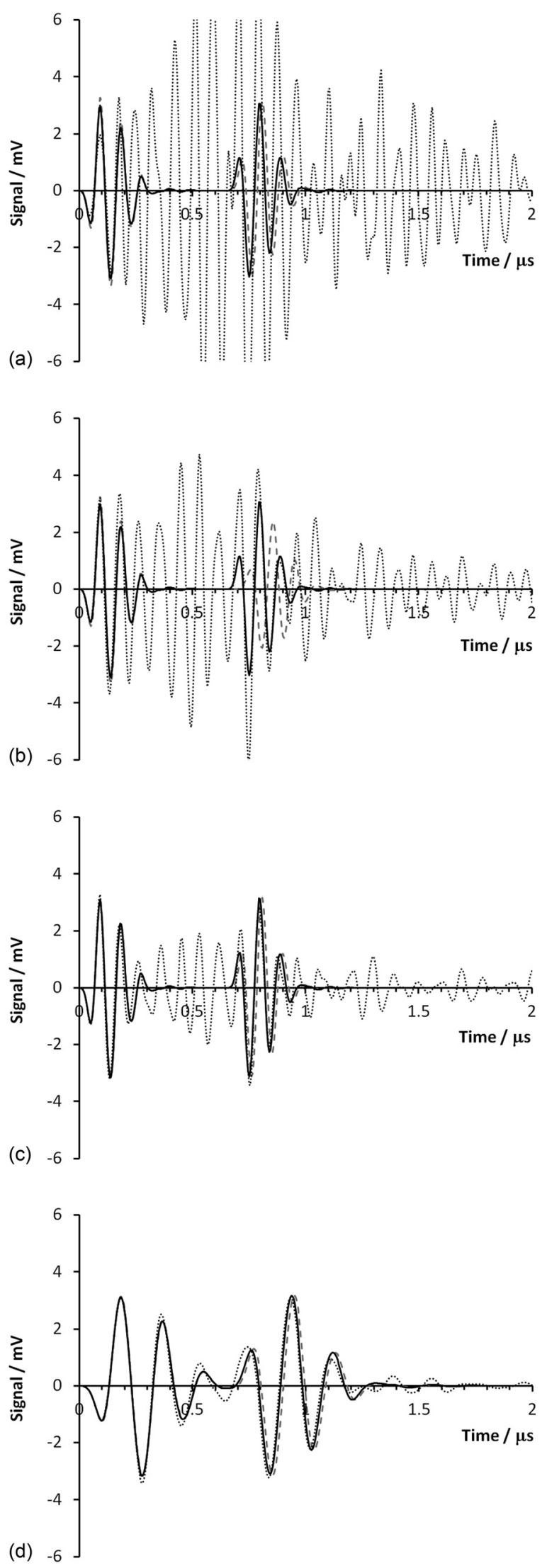

FIG. 3. Time-domain response for the effective medium model (dashed gray line), ensemble average model (solid line), and the discrete scatterer model (dotted line), all results scaled by concentration for comparison: (a) $10 \mathrm{MHz}$ center frequency transducer, $a=15.9 \mu \mathrm{m}$ cavity radius, $2 \%$ volume fraction; (b) $10 \mathrm{MHz}$ c.f. transducer, $a=15.9 \mu \mathrm{m}, 10 \%$ volume fraction; (c) $10 \mathrm{MHz}$ c.f. transducer, $a=5.0 \mu \mathrm{m}, 2 \%$ volume fraction; and (d) $5 \mathrm{MHz}$ c.f. transducer, $a=5.0 \mu \mathrm{m}, 2 \%$ volume fraction. 
correspondence between the models, and Figs. 3(c) and 3(d) demonstrate that a closer agreement is obtained when the center frequency of the transducer is lower. The combined effect of frequency and cavity radius can be seen by comparing Figs. 3(a) and 3(d), where both a reduced frequency and a smaller radius lead to the discrete scatterer model results being very close to the ensemble average and effective medium model prediction. The response becomes almost entirely due to the coherent field, and the incoherent contribution is reduced to near zero.

\section{B. Frequency-domain comparisons}

The frequency response (to a time domain impulse) of a system with $10 \mu \mathrm{m}$ cavity radius, for a range of concentrations, is shown in Fig. 4 for the three models. The plots show the absolute value of the frequency-domain response (with no transducer signal included), windowed in the timedomain to remove ripples on the frequency response caused by diffraction effects due to the truncation of the region containing cavities at a finite radial coordinate $R_{\max }$. All responses have been scaled by concentration to enable graphical comparison. Figure 4(a) shows the two reference signals from the ensemble average and effective medium models. At low frequency (low $k a$ ), the effective medium

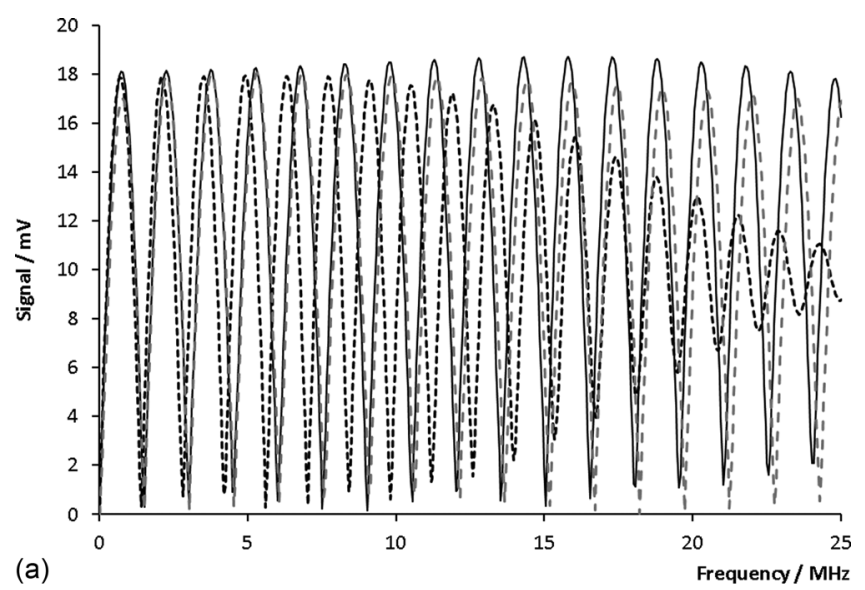

(a)

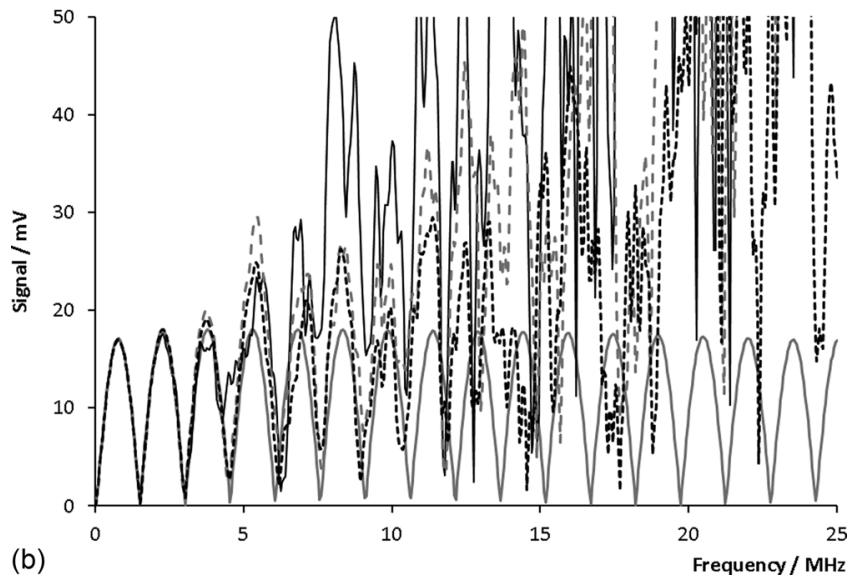

(b)

Frequency/ $\mathrm{MHz}$

FIG. 4. Frequency-domain response (absolute value) for $10.0 \mu \mathrm{m}$ cavity radius, scaled by concentration for comparison. (a) Effective medium model at $1 \%$ (dashed gray line) and 10\% (dashed black line), and the ensemble average at $1 \%$ (solid gray line) and (b) discrete scatterer model at $1 \%$ (solid black line), 5\% (dashed gray line), 10\% (dotted black line), and the ensemble average model (solid gray) at $1 \%$. model predicts a frequency response with regularly spaced peaks and pseudo-nodes, consistent with reflections from a layer, with a peak height almost invariant with frequency. For a $10 \mu \mathrm{m}$ radius, $k a=0.2$ at $10 \mathrm{MHz}$. At the higher concentration of $10 \%$ (but still at low $\mathrm{ka}$ ), the effective medium response shows a shift in the location of the pseudo-nodes due to the effect of concentration on effective wave speed. At larger $k a$ values, the effective medium response at the higher concentration of $10 \%$ reduces markedly in peak height (implying that the response is no longer proportional to concentration), and with a flattening of the response, without sharp nodes. The ensemble average response is layerlike and is similar to the effective medium response at low concentration and low $k a$. However, since the model assumes single scattering only, it is unable to reproduce the change in effective wavespeed, and therefore its peak height and node positions are independent of concentration.

Figure 4(b) shows the discrete scatterer model response compared to the ensemble average response. At low $k a$ the discrete scatterer model matches closely the ensemble average model but deviates from it increasingly as the frequency increases. At the lowest concentration, the difference is very large, with little detectable layer-like behavior in the upper frequency range, and a large amplitude variation. As the concentration increases the response approaches that of the ensemble average up to increasingly higher frequencies. At $10 \mathrm{MHz}, k d=0.9,0.5$, and 0.4 , respectively at $1 \%, 5 \%$, and $10 \%$ concentration, so a lower $k d$ produces a response closer to the ensemble average response. The single scattering assumption made by the discrete scatterer and ensemble average models means that neither can produce the features of the effective medium response seen at higher concentrations, i.e., the shift of nodes due to a change in effective speed and the non-linear change in peak height with concentration. Thus the discrete scatterer response has a mismatch in node position compared to the effective medium model, which is negligible at low concentration but increases with concentration. The frequency responses for a range of cavity radii at the same concentration show similar trends to those seen in Fig. 4(b)-increasing frequency (larger $k d$ ) causing a greater deviation between the discrete scatterer model and the ensemble average model, and a smaller cavity radius (lower $k d$ ), leading to agreement up to a higher frequency.

\section{Trends for quality of fit}

Thus far, we have identified qualitatively the trends for the agreement between a single realization of the discrete scatterer model and the corresponding ensemble average and effective medium models; these trends are now considered quantitatively. The quality of fit of the effective medium model and the ensemble average model to the discrete scatterer model results has been evaluated numerically using the sum of the squared residuals on the amplitude of the frequency response as a function of maximum frequency according to Eq. (10). Figure 5 shows the RSS as a function of frequency, with the effective medium model as the reference signal. A larger RSS indicates an increased difference between the two models, implying a less coherent response. 

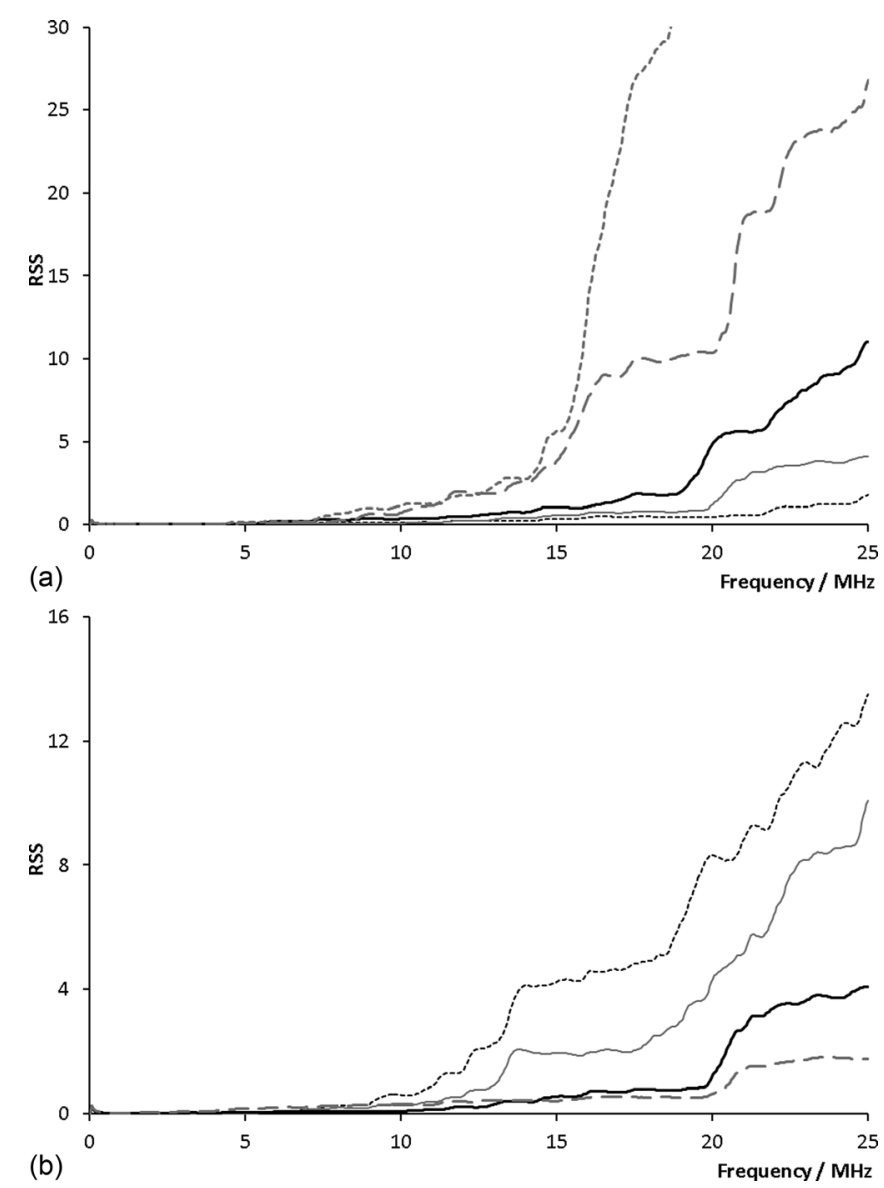

FIG. 5. Sum of squared residuals (RSS) between the discrete scatterer model and the effective medium model as a function of frequency: (a) At volume fraction of $5 \%$ for a range of cavity radii (largest radius is the top line): $5.0 \mu \mathrm{m}$ (black dotted line), $7.9 \mu \mathrm{m}$ (gray solid line), $10.0 \mu \mathrm{m}$ (black solid line), $15.9 \mu \mathrm{m}$ (gray dashed line), $20.0 \mu \mathrm{m}$ (gray dotted line); and (b) for a cavity radius of $7.9 \mu \mathrm{m}$ for a range of volume fractions (smallest volume fraction is the top line): $1 \%$ (black dotted line), $2 \%$ (gray solid line), $5 \%$ (black solid line), and 10\% (gray dashed line).

The trends observed in Secs. IV A and IV B can be identified in these plots; the RSS rises steeply with frequency, confirming that the effective medium is a better description for a single realization of scatterers at lower frequencies. Similarly, a larger cavity radius [Fig. 5(a)] and a lower concentration [Fig. 5(b)] lead to an increased difference between the two models. The trends in RSS with the ensemble average model as a reference are very similar to those shown in Fig. 5. At low frequency (below $\sim 8 \mathrm{MHz}$ ), the RSS relative to the effective medium model takes higher values as the concentration increases, due to the shift of the nodes and peaks in the frequency response caused by the change in effective wavespeed. As previously mentioned, the simplifying assumptions of the discrete scatterer and ensemble average model do not allow the prediction of this speed change and the resulting peak and node shifts; hence, a mismatch occurs between discrete scatterer and effective medium responses. However, we are concerned to monitor the deviation from layer-like effective homogeneous behavior for a single realization of scatterers, and therefore the RSS relative to the ensemble average results are of most relevance.

In order to quantify the trends with frequency, radius, and concentration, a limiting value of RSS was specified which denotes an "acceptable" agreement between the ensemble average and discrete scatterer models. Here we mean that the ensemble average model is a sufficiently accurate approximation for the signal received from a single realization of cavities, and the latter response is essentially coherent, or layer-like in character. Such a limit was established by examining the time-domain responses using the $5 \mathrm{MHz}$ center-frequency transducer; those obtained with a $10 \mathrm{MHz}$ c.f. transducer signal showed poor agreement between models for all radii and concentrations. The criteria for acceptability were: (a) close agreement for the front-face reflection; (b) minimal signal between the front and back face reflections; (c) an identifiable back-face reflection signal with similar amplitude; and (d) only small oscillations following the end of the back-face reflection. Some radius/concentration combinations showed acceptable agreement between the two models with the $5 \mathrm{MHz}$ c.f. transducer signal, and some did not. Hence an RSS limit was chosen in the frequency domain which produced similar acceptability results at $8 \mathrm{MHz}$, the $-20 \mathrm{~dB}$ bandwidth of the $5 \mathrm{MHz}$ c.f. transducer; that limit was taken to be 0.3 . This condition errs on the side of caution in terms of the use of an ensemble average model as a description of a cavity-filled region, since it accounts for a broad transducer response.

Having specified the limiting value of RSS, the frequency at which the RSS reaches that limiting value is defined as the maximum frequency for use of an effective medium model. This value is plotted as a function of radius and concentration in Fig. 6. The plots show the trends identified previously. When the cavity radius is smaller, the material behaves in a layer-like manner, as an ensemble average response up to a higher frequency than for larger cavity radii. Conversely, a larger volume fraction of cavities allows a higher frequency to be used while retaining coherent behavior.

\section{DISCUSSION}

In order to quantify the trends in the quality of fit of the ensemble average model for a single realization of scatterers, the data shown in Fig. 6 for maximum frequency $f_{\max }$ was fitted to a power law relationship of the form

$$
f_{\max } / \mathrm{MHz} \approx B a^{\alpha}(100 \phi)^{\beta},
$$

where the radius $a$ is in micrometers, resulting in exponents $\alpha=-0.71, \beta=0.32$ with a constant factor $B=36.7$. The fitted curves are shown in Fig. 6 as solid lines.

It might be expected that a coherent response (i.e., effective medium behavior) would occur from a single realization of scatterer locations when the wavelength is much greater than the length scale of the inhomogeneity in the system (the mean distance between scatterers). It has already been observed in Secs. IV A and IV B and in Figs. 3 and 4 that a greater deviation from coherence occurred for larger values of $k d$. In that case, coherence would be expected up to a maximum value of $k d$, giving exponents for the maximum frequency of $\alpha=-1, \beta=1 / 3$. The data was also fitted to this function, resulting in a constant factor $B=72.2$. The fitted 


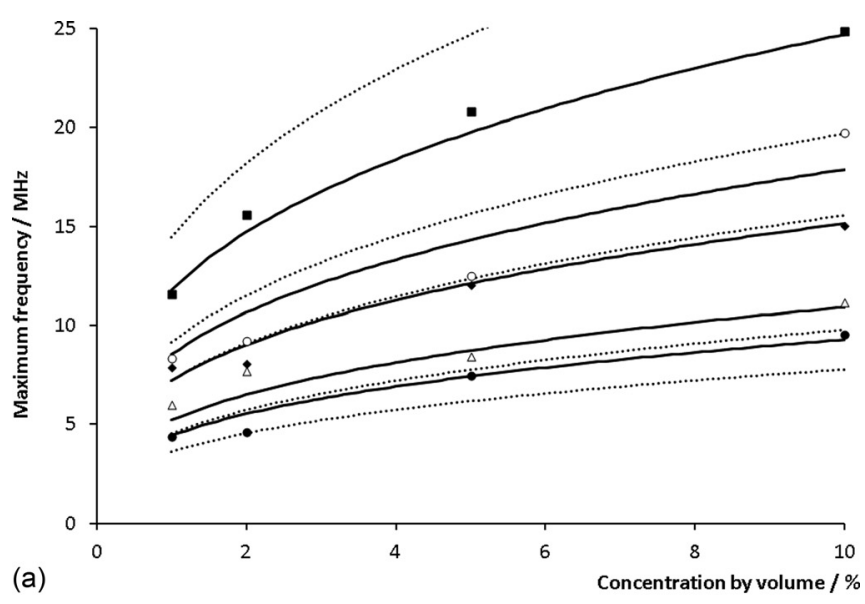

(a)

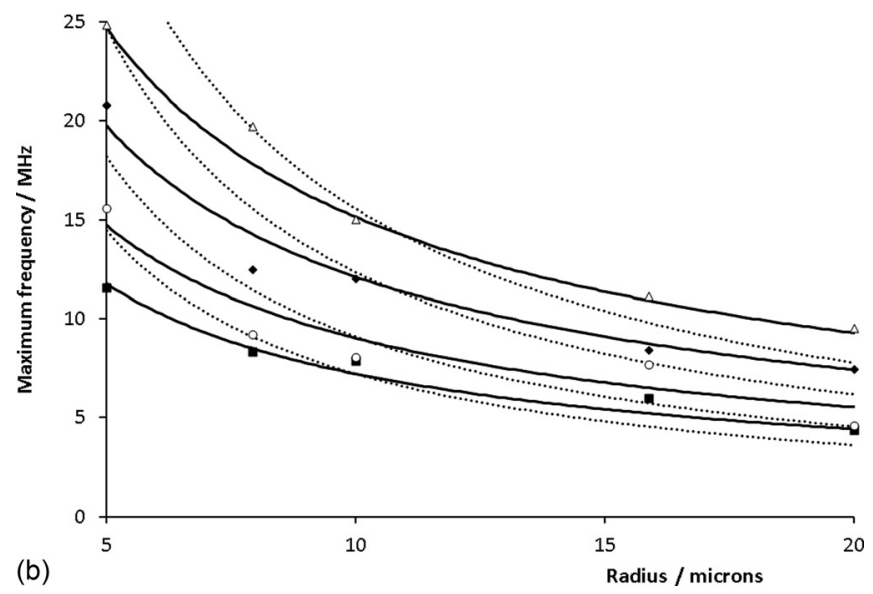

(b)

Radius / microns

FIG. 6. Maximum frequency as a function of volume fraction and cavity radius, with fitted curves: (a) Plotted against concentration for cavity radii of $5.0 \mu \mathrm{m}$ (filled squares), $7.9 \mu \mathrm{m}$ (open circles), $10.0 \mu \mathrm{m}$ (filled diamonds), $15.9 \mu \mathrm{m}$ (open triangles), and $20.0 \mu \mathrm{m}$ (filled circles); (b) plotted against radius for concentrations of $1 \%$ (filled squares), $2 \%$ (open circles), $5 \%$ (filled diamonds), and 10\% (open triangles). The solid lines are the power law with fitted coefficients, and the dotted lines are fitted according to the number density; these curves are in sequence with the top line being in (a) the smallest cavity radius and (b) the largest concentration.

curves are shown in Fig. 6 as dotted lines; they are still a reasonable fit to the data but with some difference at small radius. The fitted curves correspond to $k d=0.7$, so that the wavelength is approximately 9 times the average inter-cavity distance.

The data set examined in the present study consisted of a single realization of cavity locations for each cavity size, and trends have been fitted to these single realizations. Hence the data points plotted in Figs. 5 and 6 correspond to a single sample taken from the ensemble of possible location sets. In addition, the variability of the deviation of single realizations from the ensemble average response has been estimated by taking the standard deviation from 20 realizations at the same radius. The standard deviation in RSS was on the order of the mean RSS at the lowest frequencies, tending toward around half the mean value of RSS at higher frequencies. Thus, the deviation of a single realization from coherence can be quite variable; an estimate of the acceptable operating bandwidth for use of an effective medium interpretation would need to account for the standard deviation in the data. The results of other numerical studies ${ }^{17-21}$ which were evaluating the ensemble average (coherent) response, also indicate that the variation may be significant, since many realizations were required to achieve convergence of the coherent field.

The calculations in the present study have been derived from the field at a single point on the receiving transducer surface. As described in Sec. I, an average over a large receiving surface can be used to implement some averaging over scatterer locations, in a similar way to taking measurements at different locations or on different samples. However, the required area must sample a sufficiently large number of scatterer configurations to achieve an ensemble averaging effect. This is likely to correspond to a dimension much larger than the mean distance between scatterers. Numerical studies using a finite receiving surface area ${ }^{20,21}$ much larger than this mean distance still showed a substantial contribution from the incoherent field. The results of these studies also indicate that since many tens or hundreds of realizations of scatterer configuration were required to achieve a converged averaged reflected field, a similarly large scale-up of the receiver area would be required to achieve the same averaging effect. Therefore our study using a point receiving location is a valid method for establishing a cautious validity criterion for the effective medium description of the response from a single scatterer configuration. In our study, the response was simulated for a layer, taken as a large but finite region, with a lateral dimension much larger than the mean distance between scatterers; hence, some ensemble-averaging may already occur in the responses.

\section{CONCLUSIONS}

The investigation reported in this paper has demonstrated the conditions under which the backscattered (reflected) response to a compressional wave of a single configuration of locations of spherical cavities in a solid approaches the coherent field, i.e., that obtained from a homogeneous layer with ensemble-averaged properties. Under such conditions, an effective medium model is a good representation of the response from a single sample. By numerical simulation and fitted trends for the frequency response, we have established that the effective medium model is a better fit at lower frequencies, smaller cavity radii, and a higher volume fraction of cavities. The results are consistent with the requirement that the wavelength is much larger than the average distance between cavities, corresponding to at least nine cavities per wavelength.

What remains to be established is the effect of averaging over a finite receiving area (rather than a single point), and the mean and variance of the incoherent response for different scatterer configurations. In addition, since focusing techniques are often used in NDE applications, it would be of interest to study the response from a small focal region containing cavities, rather than a layer. Correlations in scatterer locations were also not considered in the present study, although some work exists on the effect of such correlations on the expected response, ${ }^{12}$ and this could be a further relevant area for investigation.

The significance of the work reported here is that it enables the use of effective medium properties in modeling 
ultrasonic wave propagation in composites, in particular for porosity flaws. The results of this paper demonstrate the conditions under which the use of effective medium properties is justified. Typical cavity size and volume fraction observed in composites fall within those conditions.

\section{ACKNOWLEDGMENT}

This work was in part funded by the UK Engineering and Physical Sciences Research Council (EPSRC) through the UK Research Centre for Non-Destructive Evaluation (RCNDE).

${ }^{1}$ R. E. Challis, M. J. W. Povey, M. L. Mather, and A. K. Holmes, "Ultrasound techniques for characterizing colloidal dispersions," Rep. Prog. Phys. 68(7), 1541-1637 (2005).

${ }^{2}$ C. M. Linton and P. A. Martin, "Multiple scattering by multiple spheres: A new proof of the Lloyd-Berry formula for the effective wavenumber," SIAM J. Appl. Math. 66(5), 1649-1668 (2006).

${ }^{3}$ P. A. Martin, A. Maurel, and W. J. Parnell, "Estimating the dynamic effective mass density of random composites," J. Acoust. Soc. Am. 128(2), 571-577 (2010).

${ }^{4}$ C. Aristegui and Y. C. Angel, "Coherent sound propagation across the effective interfaces of an immersed screen," Wave Motion 47, 199-204 (2010).

${ }^{5}$ J.-Y. Kim, "Models for wave propagation in two-dimensional random composites: a comparative study,” J. Acoust. Soc. Am. 127(4), 2201-2209 (2010).

${ }^{6}$ W. J. Parnell, I. D. Abrahams, and P. R. Brazier-Smith, "Effective properties of a composite half-space: exploring the relationship between homogenization and multiple scattering theories," Q. J. Mech. Appl. Math. 63(1), 1-32 (2010).

${ }^{7}$ V. J. Pinfield, R. E. Challis, and R. A. Smith, "A comparison of stochastic and effective medium approaches to the backscattered signal from a porous layer in a solid matrix," J. Acoust. Soc. Am. 130(1), 122-134 (2011).

${ }^{8}$ S. K. Kanaun and V. M. Levin, "Effective medium method in the problem of axial elastic shear wave propagation through fiber composites," Int. J. Solids Struct. 40, 4859-4878 (2003).

${ }^{9}$ P. J. Li, Q. Z. Wang, and S. F. Shi, "Differential scheme for the effective elastic properties of nano-particle composites with interface effect," Comput. Mat. Sci. 50(11), 3230-3237 (2011).

${ }^{10}$ S. K. S. Kanaun and E. Pervago, "Combining self-consistent and numerical methods for the calculation of elastic fields and effective properties of 3D-matrix composites with periodic and random microstructures," Int. J. Eng. Sci. 49(5), 420-442 (2011).
${ }^{11}$ J. R. Willis, "Effective constitutive relations for waves in composites and metamaterials," Philos. Trans. R. Soc. London, Ser. A 467(2131), 18651879 (2011)

${ }^{12}$ V. Leroy, A. Strybulevych, J. H. Page, and M. G. Scanlon, "Influence of positional correlations on the propagation of waves in a complex medium with polydisperse resonant scatterers," Phys. Rev. E 83(4), 046605 (2011).

${ }^{13}$ L. L. Foldy, "The multiple scattering of waves," Phys. Rev. 67(3-4), 107119 (1945).

${ }^{14} \mathrm{P}$. C. Waterman and R. Truell, "Multiple scattering of waves," J. Math. Phys. 2, 512-537 (1961).

${ }^{15}$ M. Lax, "Multiple scattering of waves," Rev. Mod. Phys. 23, 287-310 (1951).

${ }^{16}$ J. M. Conoir and A. N. Norris, "Effective wavenumbers and reflection coefficients for an elastic medium containing random configurations of cylindrical scatterers," Wave Motion 47(3), 183-197 (2010).

${ }^{17}$ J. Dubois, C. Aristegui, O. Poncelet, and A. L. Shuvalov, "Coherent acoustic response of a screen containing a random distribution of scatterers: comparison between different approaches," J. Phys.: Conf. Ser. 269, 012004 (2011).

${ }^{18}$ M. Ostoja-Starzewski, "Microstructural randomness versus representative volume element in thermomechanics," J. Appl. Mech. 69(1), 25-35 (2002).

${ }^{19}$ M. Ostoja-Starzewski, "Material spatial randomness: From statistical to representative volume element," Probab. Eng. Mech. 21(2), 112-132 (2006).

${ }^{20}$ A. Maurel, "Reflection and transmission by a slab with randomly distributed isotropic point scatterers," J. Comput. Appl. Math. 234, 1842-1850 (2010).

${ }^{21}$ B. Galaz, G. Haiat, R. Berti, N. Taulier, J. J. Amman, and W. Urbach, "Experimental validation of a time domain simulation of high frequency ultrasonic propagation in a suspension of rigid particles," J. Acoust. Soc. Am. 127(1), 148-154 (2010).

${ }^{22}$ J. W. S. Strutt (Baron Rayleigh), The Theory of Sound, Reprint of 1894, 2nd ed. (Dover Publications, New York, 1945), Chap. XVII, pp. 272-284.

${ }^{23}$ C. F. Ying and R. Truell, "Scattering of a plane longitudinal wave by a spherical obstacle in an isotropically elastic solid," J. Appl. Phys. 27, 1086-1097 (1956).

${ }^{24}$ R. E. Challis, J. S. Tebbutt, and A. K. Holmes, "Equivalence between three scattering formulations for ultrasonic wave propagation in particulate mixtures," J. Phys. D: Appl. Phys. 31(24), 3481-3497 (1998).

${ }^{25}$ Y. C. Angel and C. Aristegui, "Analysis of sound propagation in a fluid through a screen of scatterers," J. Acoust. Soc. Am. 118(1), 72-82 (2005).

${ }^{26} \mathrm{~F}$. Luppe and J. M. Conoir, "Multiple scattering by cylinders randomly located in a fluid: Effective properties," J. Phys.: Conf. Ser. 269, 012015 (2011).

${ }^{27}$ MATLAB ${ }^{\circledR}$ version R2008a (v. 7.6.0.324), The Mathworks Inc., Natick, MA. 